\title{
Adaptive Enhancement Techniques for Solar Images
}

\author{
Mohammad A. A. Al-Rababah ${ }^{1}$, Abdusamad Al-Marghilani ${ }^{1}$, Mohammed M. Al-Shomrani' ${ }^{2}$, \\ Ibrahim A. Atoum ${ }^{3}$
}

${ }^{1}$ Northern Border University, Arar, KSA; ${ }^{2}$ King Abdulaziz University, Jeddah, KSA; ${ }^{3}$ Hail University, Hail, KSA.

Email: dsmadi@rambler.ru

Received July $23^{\text {rd }}, 2013$; revised August $23^{\text {rd }}, 2013$; accepted August $31^{\text {st }}, 2013$

Copyright (C) 2013 Mohammad A. A. Al-Rababah et al. This is an open access article distributed under the Creative Commons Attribution License, which permits unrestricted use, distribution, and reproduction in any medium, provided the original work is properly cited.

\begin{abstract}
Radio astronomy radio telescope plays the role of a linear operator, affecting the function that describes the object of research, formation of image of a monitored object. This paper presents methods for reconstruction and correction of solar radio images using the algorithm of rejections, the updated Weiner-filter, and the method CLEAN designed by Hëgbomom (Pseudonym, 2009) for point sources. It is the process of numerical convolution in signal handling, an algorithm for separating weak-contrast formations on the solar which represents most points of the actual limb by using the ellipse equation. Consequently, the filling algorithm is applied by moving from the center to the ellipse points and filling each point by solar image data. Finally, a linear limb-darkening expression is used to remove the limb darkening. Different examples of the intermediate and final results are presented in addition to the developed algorithm.
\end{abstract}

Keywords: Image Processing; Solar Imaging; Image Enhancement; Linear Transformation Functions; Limb Darkening; Solar Disk; Limb Fitting

\section{Introduction}

At the present day the problem of object detection on digital picture is very challenging due to rapid development of photo and video electronics. Despite the fact that physical reality contains a lot of different objects the development of detection algorithm for a narrower class of objects - human faces - is of considerable interest [1]. This is due to the increasing degree of automation of various processes and production systems. The particular application of the algorithm of human faces detection may be as follows: automatic registration of visitor's number in the supermarkets and entertainment [2].

Centres crossing control systems in various institutions, airports, subway; automated systems to prevent accidents, they monitor the face of vehicle's driver; man-machine intelligent interfaces, current demands in establishment of such systems impose strict limits on the speed of the algorithms executions, which shall operate in real time mode. Hence, the perspective problem to be solved is creation of fast and reliable algorithm of human faces detection.

Available approaches are to face the detection problem. In the last ten years, the dynamic elaborations are conducted in the field of images detection and there were offered a variety of detection methods: the method of principal components, methods with using of histograms, neural network, Bayesian networks, statistical methods, etc. Some of these detecting algorithms are invariant ones with respect to the object, while others use such a priori knowledge about the object, like the shape, colours, relative position of parts [3].

In computer graphics, image enhancement is one class of five different classes of digital image processing operations. These classes represent the fundamental techniques of this field. An image enhancement operation seeks to improve the quality of a digitally stored image by manipulating the image with software. This operation may be an end itself or could be used as a preprocessing phase to ease the next-processing step or as postprocessing steps to improve the visual perception of a processed image. Some images need an easy enhancement operation while other images need more sophisticated operations. One of these later images is solar images. These images have to be pre-processed in order to correct them for geometrical or photometric distortions.

One of the initial efforts in this area is the standardization techniques. 


\section{Developed by Solar Images}

One of the most successful recovery procedures is cleaning algorithm (CLEAN), developed for point sources. It is the process of numerical convolution in signal handling. Sizes, requiring many thousands of method of cleansing, to stabilize and reduce the distorting effects: cleaning, stabilized anti-aliasing and cleaning method of cleaning, stabilized intrude. Low value enhancing algorithm downward distortion is efficient for cleaning, stabilized anti-aliasing at the asymptotic behavior of the process. In addition, it is worth noting that the second methodology cleaning, stabilized intrude cannot be practically used, because the process does not always converge [4].

For feature selection for each iteration of the loop method is used. Valuable qualities are especially noticeable when processing algorithm of extended areas, a brief look at a classical method. Look at the Equation (1) in the one-dimensional version:

$$
\begin{aligned}
& g(x)=\int h\left(x-x_{i}\right) f\left(x_{i}\right) \mathrm{d} x_{i} \\
& f(x)=\sum_{k=1}^{\infty} p(k) \delta\left(x-x_{k}\right),
\end{aligned}
$$

where $p(k)$ is a function of the coefficients the Equation (2)

$$
\begin{aligned}
g(x) & =\sum_{i=1} h\left(x-x_{i 1}\right) f\left(x_{i 1}\right) \\
& =\sum_{i=1} h\left(x-x_{i 1}\right) x \sum_{k=1}^{\infty} p(k) \delta\left(x_{1 i}-x_{k}\right) \\
& =\sum_{k=1}^{\infty} p(k) \sum_{i=1}^{N}\left(h(x)-x_{i 1}\right) \delta\left(x_{1 i}-x_{k}\right) \\
& =\sum_{k=1}^{\infty} p(k) h\left(x-x_{k}\right)
\end{aligned}
$$

From here you can see that the function $g(x)$ is visualized as a sum of an infinite number of functionsoriented diagrams. The function $g(x)$ is called "Dirty" card, a function $h(x)$-"dirty beam". From these considerations it should be purely empirical algorithm associated with the subtract join dirty beam from a dirty cards distribution granularity the brightness on the replies from point sources, and then replacing each of them for the response of the "pure".

1) The most vivid picture element $(a)$ in a distorted image, such that the element of $1, m j, k a 1$ all $>\neq j$ and $\mathrm{m} \neq k$.

2) A new refined image is PD-1, the Equation (3)

$$
\begin{aligned}
& f c_{1, m} \tau a_{j, k} \delta_{i-j, m-k} \rightarrow f c_{i, m} \\
& a_{i, m}-\tau a_{j, k} \sum_{\Omega h(i, m)} h_{i-j, m-k} \rightarrow a_{i, m^{\prime}} \\
& \tau<1 .
\end{aligned}
$$

Refined image is collapsed with the perfect beam of $h(x)$, with the main lobe and strongly reduced side, the main advantage of the method is its simplicity. Negative.

The quality of the algorithm is a small calculation speed and apparent distortions in the form of wrinkles and "flings" structure, which manifests itself in the extended areas. It is worth mentioning that the algorithm does not contain clear criteria for selecting options increase [5].

\section{Material and Methods}

Geometrical model of thin layer matting model to produce a quantitatively collation of the darkening of the Sun to the edge of the visible wavelength range is a thin layer of emitting model. According to this model, the entire visible spectrum of the Sun is emitted at a certain depth $H_{V I S}$ Therefore, Figure 1 model to produce a quantitative calculation.

By known dependencies $T(l), B(T)$ radial temperature distribution and function respectively source can build the intensity of radiation output the Equation (4).

$$
I(r, H)=\frac{B\left(T\left(1-\sqrt{1+H_{V I S}{ }^{2}-2 H_{V I S} \sqrt{1+r^{2}}}\right)\right)}{B\left(T\left(H_{V I S}\right)\right)} .
$$

Model of emitting layer final thickness.

In this case the value normalized to 1 the intensity is determined by the integration of line of sight and an expression $s-s^{\prime}$ of the beam the Equation (5)

$$
I(r, H)=\frac{\int_{0}^{H} B(T(h(l, r))) \alpha(h(l, r)) \mathrm{d} l}{\int_{0}^{H} B(T(l)) \alpha(l) \mathrm{d} l}
$$

$$
B, T, \alpha
$$

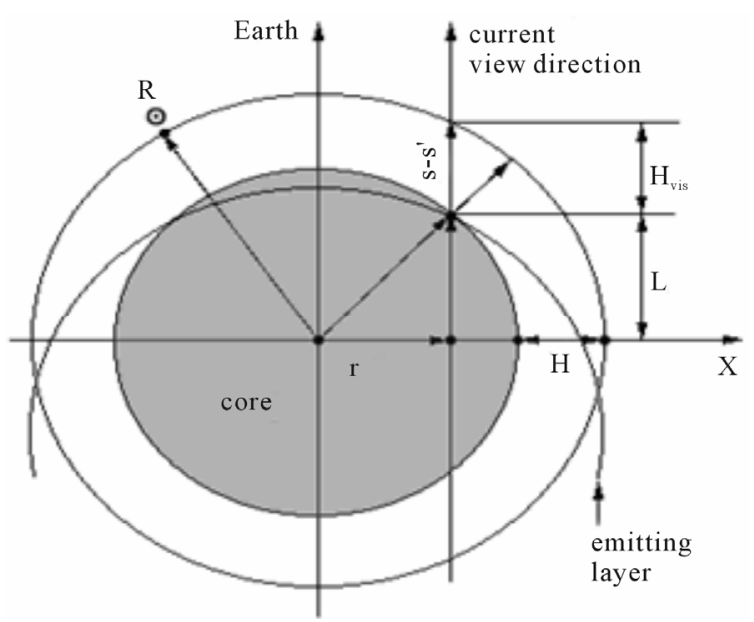

Figure 1. Radial slices of sun-illustration to describe geometric models of the atmosphere. 


$$
h(l, r)=1-\sqrt{1+l^{2}-2 l \sqrt{1+r^{2}}}
$$

In the assumption of equilibrium nature of the processes $B(T) \sim T^{4}$ involved. The possibility of such a deal may be justified, by calculating the Equation (7).

$$
C=\int_{v_{0}}^{\infty} \alpha_{v} \mathrm{~J}_{v} \frac{\mathrm{d} v}{h v} / \int_{v_{0}}^{\infty} \alpha_{v} \mathrm{~B}_{v} \frac{\mathrm{d} v}{h v}
$$

The ratio of the radiant flux to the average number of a volume. For the Sun compared $C$ the value negligibly small.

Grey model of the atmosphere $\kappa_{v}=\bar{\kappa}$ does not depend on the equation we obtain the integral equation of linear on the singular kernel the Equation (8).

$$
\begin{gathered}
(B(\tau)=c[\tau+q(\tau)]) \\
q(\tau)=q_{\infty}-\frac{F}{8} \int_{0}^{1} \frac{\exp (-\tau / \mu) \mathrm{d} \mu}{I(0, \mu)\left[(1-\mu \operatorname{Arth} \mu)^{2}+\left(\frac{\pi \mu}{2}\right)^{2}\right]}, \\
q_{\infty}=\frac{6}{\pi^{2}}+\frac{1}{\pi} \int_{0}^{\pi / 2}\left(\frac{3}{\theta^{3}}-\frac{1}{1-\theta \operatorname{ctg}(\theta)}\right) \mathrm{d} \theta \\
I(0, \mu)=\int_{0}^{\infty} B(t) \mathrm{e}^{-t / \mu} \frac{\mathrm{d} t}{\mu}=\frac{\sqrt{3}}{4} F(1+\mu) \\
\exp \left\{\frac{\mu}{\pi} \int_{0}^{\pi / 2} \frac{\ln \left(\frac{\sin ^{2} \theta}{1-\theta \operatorname{ctg}^{2} \theta}\right)}{\cos ^{2} \theta+\mu^{2} \sin ^{2} \theta} \mathrm{d} \theta\right.
\end{gathered}
$$

\section{Erosion and Dilation}

Almost all types of solar activity very effective evident in the microwave the radiation of the Sun. Monitoring of solar active-Ness, including the faint-events systematically runs from sunrise to sunset The Sun against the background of the solar disk in microwave-PTO radiation with high spatial and temporal resolution, the weakly contrasting formations contain valuable information needed to examine the conditions of emergence, peculiarities of evolution and prediction of solar-terrestrial interaction. When identifying physical properties of Coronal holes, prominences and filaments important identification of these entities on a solar disk. In the microwave radiation it is difficult. Proposed earlier identification tools require observation of the Sun with high spatial resolution on two or more wavelengths, which is not always possible. The Sun takes place in several stages. At the first preliminary processing phase noise filtering produced: 1) Filters, remove obstacles; That linked to the inaccuracy of the calibration coefficient gain and zero level channels; 2)
Median filter for eliminating pulse noise; 3) Low frequency filter for removing noise outside the bandwidth to In general, a recursive filter was used. In the task of identification of fibers, which are typically an elongated East-West area, type of filter applied, obviously, the bigger the SE size is, the longer the time spent by the computer calculating all the values will be. This part of the detection is the most time consuming in the computational process. Figure 2 summarizes the whole process $[6,7]$.

Initially, the exponential function is expressed and computed for every intensity value that is less than an empirical value $(\alpha)$, otherwise express and calculate the logarithmic value for the underlying intensity value. Suppose we have any two positive real numbers $a$ and $b$ then calculates the value of $m$ as in Equation $(12)[8,9]$.

$$
m=a \log (1+m b)
$$

where $m$ is the maximum intensity value in the underlying image (255). Then calculate $b$ as in Equation (13):

$$
b=\frac{1-\exp \left(\frac{m}{a}\right)}{m}
$$

Given a constant $\alpha$, pixels in original image $O(i, j)$ are

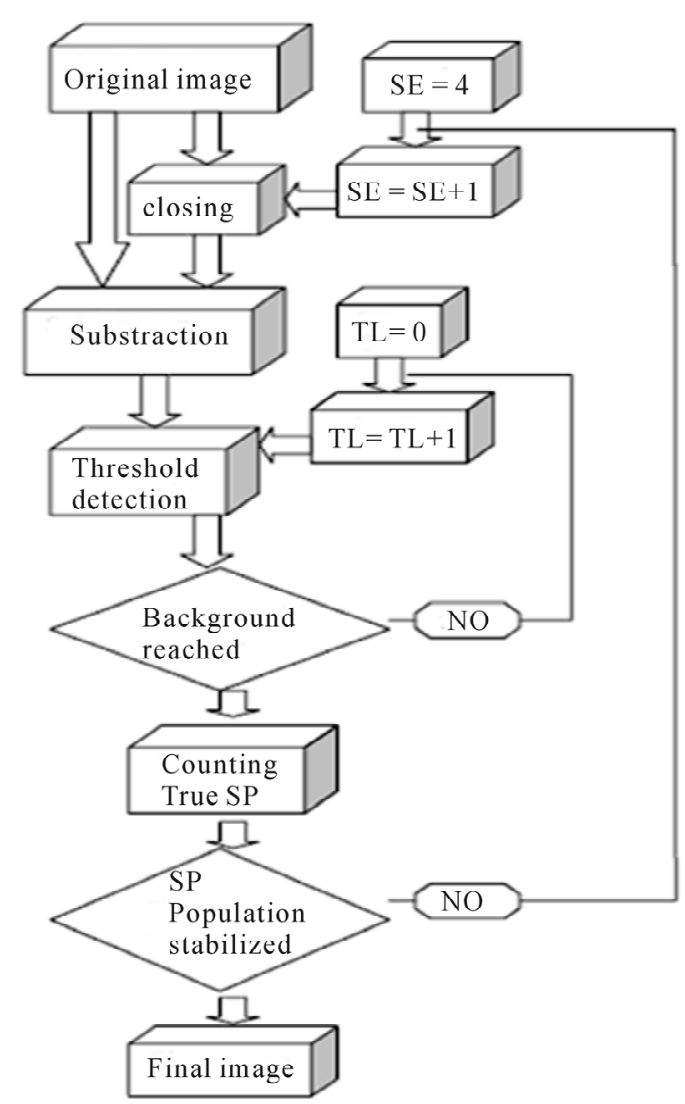

Figure 2. Chart diagram of the sunspot detection procedure. 
modified as in Equation (3) to obtain the required enhanced image $E(i, j)$ :

If $O(i, j)<\alpha$, then

$$
E(i, j)=a \log [1+b O(i, j)]
$$

else

$$
E(i, j)=\left[\exp \left[\frac{o(i, j)}{a}\right]-1\right] / b
$$

The value of beta $(\beta)$ was empirically found to be 10 , whereas the alpha $(\alpha)$ value is 100 and $a=1000$. The algorithm has the effect to remove the background and reside the foreground $[9,10]$ the result of this is shown in Figure 3.

\section{Discussion and Result}

Where the semi-major axis and $b$ is is the semi-minor axis, as illustrated in Figure 4.

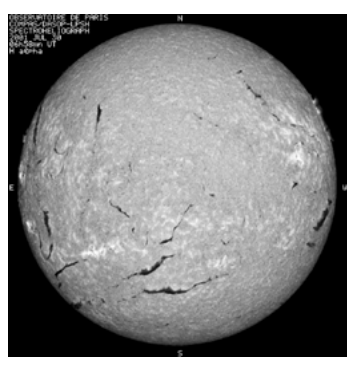

(a)

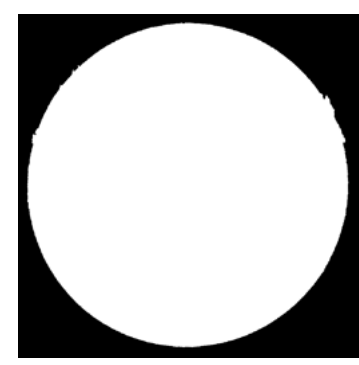

(b)
Figure 3. Results of detecting the solar disk. (a) The original solar image observed at Meudon Observatory on 31/7/ 2001; (b) The solar disk detected.

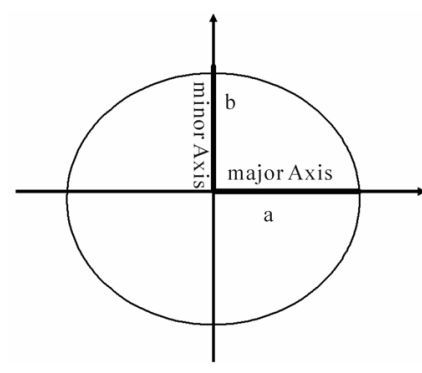

(a)

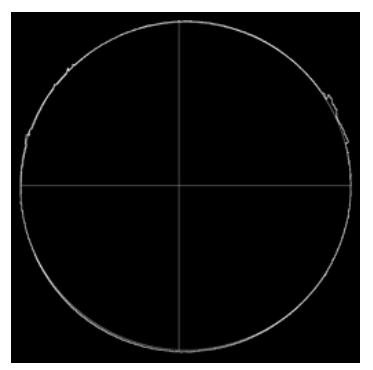

(b)

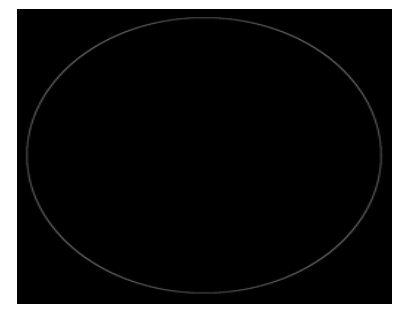

(c)

Figure 4. (a) Semi major and minor axis; (b) The actual limb and the ellipse drawn; (c) The ellipse after removing the actual limb point.

\section{Filling the Ellipse}

The filling step was designed to make a distinction between the solar object region and the background region that to be positioned outside the solar object. It is applied by moving from the candidate center points toward the ellipse points and filling every point inside the ellipse and not related to the limb by the actual solar image pixels. Figure 5 shows the ellipse after filling it with the solar disk data $[10,11]$.

\section{Limb Darkening Removal}

The sun is not equally bright all over, but it is darkened towards the limb, it is a phenomenon called limb darkening which should be calculated and removed. A sensible empirical representation of the limb darkening is governed by the linear limb-darkening expression shown in Equation (5) [12].

$$
\frac{I(\mu)}{I(1)}=1-u(1-\mu)
$$

$I$ (1) is the specific intensity at the center of the disk, $\mathrm{u}$ is the LDC (Limb-arkening coefficient), it's empirically found to be equal 0.5 , and $\mu=\cos (\theta)$, where $\theta$ is the angle between the line of sight and the emergent intensity. Figure 6(a) shows an original solar image and Figure 6(b) shows it after limb-darkening removed. These enhanced images could be used then for the post-processing applications like detecting and tracking [13,14].

Solar features as shown in Figure 7. Figure 7(a) shows an enhanced image and Figure 7(b) shows the image after applying an adaptive thresholding technique for detecting solar filament.

\section{Conclusions}

This presented work was to detect a cleaned solar disk as precisely as possible that could be used for different techniques of solar image analysis. Different tools were developed in this paper to achieve this goal initially by detecting the solar disk and removing the background spots by using linear exponential and logarithmic func-

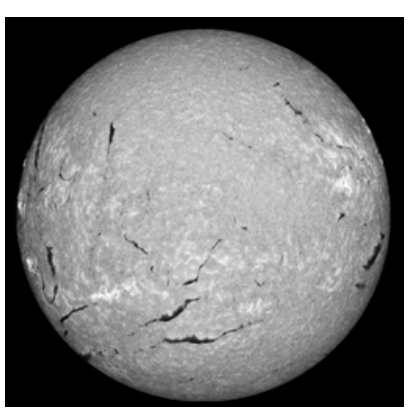

Figure 5. Filled solar disk for solar image observed at Meudon Observatory on 31/7/2001. 


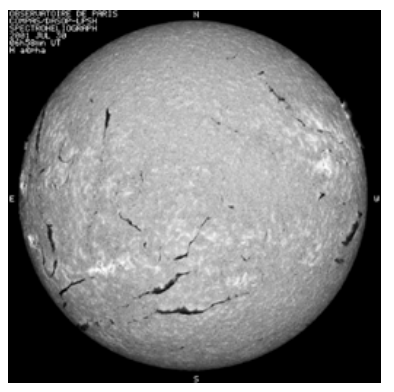

(a)

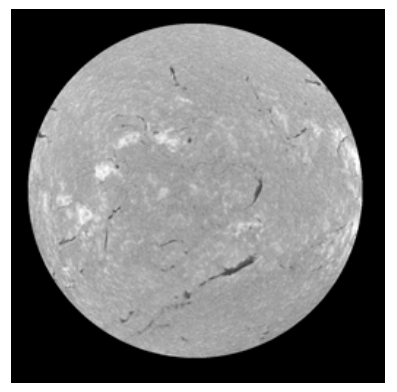

(b)
Figure 6. (a) Original image observed at Meudon Observatory on 1/04/2002; (b) The result removing limb darkening.

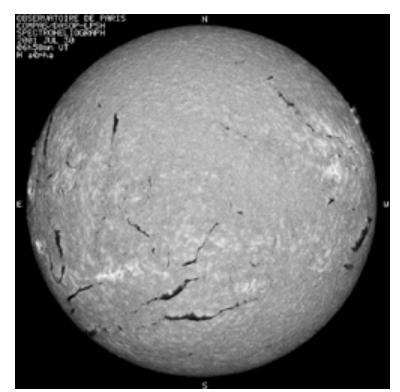

(a)

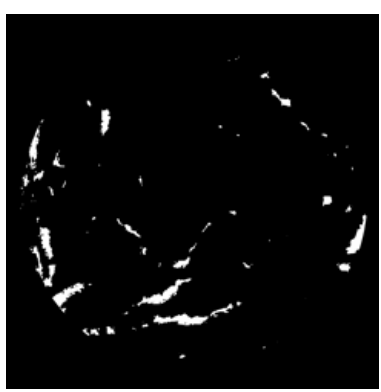

(b)
Figure 7. (a) An original Image observed at Meudon Observatory on 31/7/2001; (b) The result after applying ALT.

tions. Then, we determine the solar limb by using morphological operations.

This gives the chance to determine the initial estimation of the solar disk radius and center. Thereafter, using an elliptical equation, the elliptical shape of the solar disk is drawn which approximately includes most of the initial estimations of the solar limb. This process is followed by filling the elliptical shape with the actual solar disk data, and finally removing the limb darkening. Regardless of this progress, there are still some challenges that are not solved.

\section{REFERENCES}

[1] V. V. Zharkova, S. S. Ipson, S. I. Zharakov, A. Benkhalil, J. Aboudarham and R. D. Bentley, "A Full-Disk Image Standardisation of the Synoptic Solar Observations at the Meudon Observatory," Solar Physics, Vol. 214, No. 1, 2003, pp. 89-105. http://dx.doi.org/10.1023/A:1024081931946

[2] N. Fuller and J. Aboudarham, "Automatic Detection of Solar Filaments versus Manual Digitization," International Conference on Knowledge-Based and Intelligent Information \& Engineering Systems, Vol. 3215, 2004, pp.
467-475.

http://dx.doi.org/10.1007/978-3-540-30134-9 63

[3] N. Fuller, J. Aboudarham and R. D. Bentley, "Filament Recognition and Image Cleaning on Meudon $\mathrm{H} \alpha \mathrm{Spec}-$ troheliograms," Solar Physics, Vol. 227, No. 1, 2005, pp. 61-73. http://dx.doi.org/10.1007/s11207-005-8364-1

[4] M. Qu, F. Y. Shih, J. Jing and H. Wang, "Automatic Solar Filament Detection Using Image Processing Techniques," Solar Physics, Vol. 228, No. 1-2, 2005, pp. 119135. http://dx.doi.org/10.1007/s11207-005-5780-1

[5] F. Y. Shih and A. J. Kowalski, "Automatic Extraction of Filaments in H-Alpha Solar Images," Solar Physics, Vol. 218, No. 1-2, 2003, pp. 99-122. http://dx.doi.org/10.1023/B:SOLA.0000013052.34180.58

[6] P. N. Bernasconi, D. M. Rust and D. Hakim, “Advanced Automated Solar Filament Detection and Characterization Code: Description, Performance and Results," Solar Physics, Vol. 228, No. 1-2, 2005, pp. 97-119. http://dx.doi.org/10.1007/s11207-005-2766-y

[7] R. Qahwaji and T. Colak, "Automatic Detection and Verification of Solar Features," International Journal of Imaging Systems and Technology, Vol. 15, 2005, pp. 199210. http://dx.doi.org/10.1002/ima.20053

[8] A. D. Joshi, N. Srivastava and S. K. Mathew, "Automated Detection of Filaments and Their Disappearance Using Full-Disc H $\alpha$ Images," Solar Physics, Vol. 262, No. 2, 2009, pp. 425-436. http://dx.doi.org/10.1007/s11207-010-9528-1

[9] Y. Yuan, F. Y. Shih, J. Jing, H. Wang and J. Chae, "Automatic Solar Filament Segmentation and Characterization," Solar Physics, Vol. 272, No. 1, 2010, pp. 101-117. http://dx.doi.org/10.1007/s11207-011-9798-2

[10] National Solar Observatory, "Ca K and H Alpha Images Explained," 1996.

http://eo.nso.edu/MrSunspot/answerbook/cak_ha_expl.html

[11] G. Kom, A. Tiedeu and M. Kom, "Automated Detection of Masses in Mammograms by Local Adaptive Thresholding," Computers in Biology and Medicine, Vol. 37, No. 1, 2007, pp. 37-48. http://dx.doi.org/10.1016/j.compbiomed.2005.12.004

[12] N. Efford, "Digital Image Processing, a Practical Introduction Using Java," 1st Edition, Pearson Education Limited, Addison Wesley, 2009.

[13] A. M. K. Hamid, "Linearized Limb-Darkening Coefficients for Use in Analysis of Eclipsing Binary Light Curves," Astrophysics and Space Science Journal, Vol. 53, No. 1, 1977, pp. 181-192.

[14] I. A. Atoum, R. S. Qahwaji, T. Colak and Z. H. Ahmed, "Adaptive Thresholding Technique for Solar Filament Segmentation," Ubiquitous Computing and Communication Journal, Vol. 4, 2009, pp. 91-95. 